\title{
Consequences of Mastoidectomy on Bone Conducted Sound Based on Simulations in a Whole Human Head
}

Srdan Prodanovic and Stefan Stenfelt

The self-archived postprint version of this journal article is available at Linköping University Institutional Repository (DiVA):

http://urn.kb.se/resolve?urn=urn:nbn:se:liu:diva-171677

N.B.: When citing this work, cite the original publication.

Prodanovic, S., Stenfelt, S., (2020), Consequences of Mastoidectomy on Bone Conducted Sound Based on Simulations in a Whole Human Head, Otology and Neurotology, 41(9), E1158-E1166.

https://doi.org/10.1097/MAO.0000000000002748

Original publication available at:

https://doi.org/10.1097/MAO.0000000000002748

Copyright: Lippincott, Williams \& Wilkins

http://www.lww.com/ 
Title:

Consequences of mastoidectomy on bone conducted sound based on simulations in a whole human head

Running Head:

Mastoidectomy effects on bone conduction

Authors:

Srdjan Prodanovic, Department of Clinical and Experimental Medicine, Linköping University, Sweden

Stefan Stenfelt, Department of Clinical and Experimental Medicine, Linköping University, Sweden

Corresponding author:

Srdjan Prodanovic, Department of Biomedical and Clinical Sciences, Linköping University, Linköping 581 85, Sweden.

Email: srdan.prodanovic@liu.se

Conflicts of Interest and Source of Funding:

Swedish hearing research foundation (2017-557)

The authors declare no conflict of interest.

Key Words:

bone conduction, LiUHead, mastoidectomy, finite element model 


\section{ABSTRACT}

Hypothesis: The aim of this study is to investigate how a mastoidectomy surgery affects bone conduction $(\mathrm{BC})$ sound transmission using a whole head finite element model.

Background: Air conduction $(A C)$ and $B C$ hearing thresholds are normally used to evaluate the effect of an ear surgery. It is then assumed that the $B C$ hearing thresholds are unaffected by the surgery. Moreover, BC hearing aids are used in cases of unilateral or conductive hearing loss in heads that have undergone a mastoidectomy surgery. Given the invasiveness of the surgery, the $\mathrm{BC}$ hearing sensitivity may be altered by the surgery itself.

Methods: Two types of mastoid surgery, canal wall up and canal wall down, with and without obliteration, were simulated in a whole head finite element model for BC stimulation, the LiUHead. The evaluations were conducted for two different methods of applying the $\mathrm{BC}$ sound, at the skin surface (B71 transducer) and directly at the bone (BC hearing aid).

Results: The results showed that a mastoidectomy surgery increased the cochlear vibration responses with $\mathrm{BC}$ stimulation. The increase was less than $5 \mathrm{~dB}$, except for a canal wall down surgery which gave an increase of up to $10 \mathrm{~dB}$ at frequencies close to $10 \mathrm{kHz}$. The increase was greater at the ipsilateral cochlea compared to the contralateral cochlea.

Conclusion: A mastoidectomy surgery increases the vibration at both cochleae for $\mathrm{BC}$ stimulation and the increase generally improved with frequency. Obliteration of the surgical cavity does not influence BC sound transmission. 


\section{INTRODUCTION}

Bone conduction $(\mathrm{BC})$ is defined as the sound transmission to the inner ear through vibrations of the skull bone and surrounding tissues $(1,2)$. $B C$ is a parallel pathway to air conduction $(A C)$, where the sound is transmitted through air. For successful $A C$, the outer and middle ear need to be intact. BC hearing does not require a normal outer and/or middle ear, even though both have been recognized as pathways for $B C$ sound perception (3-5). For this reason, $B C$ together with $A C$ testing is used for the diagnosis of a middle ear lesion. Moreover, because $B C$ hearing is largely unaffected by the outer and middle ear status, $\mathrm{BC}$ hearing aids are used for hearing rehabilitation in the case of conductive hearing loss, when a conventional hearing aid device cannot be used (6-8). BC hearing aids are especially useful in cases of bilateral conductive hearing loss where binaural hearing can be recovered (9-11).

BC stimulation is applied directly on the skull bone or to the skin (12). An example of the former is the bone anchored hearing aid (BAHA), where a titanium screw is implanted in the skull bone around $55 \mathrm{~mm}$ behind the ear canal opening. A typical device from the latter group is the audiometric $B C$ transducer B71 (or B81) (Radioear corp. USA), which applies the stimulation via a $\varnothing 15 \mathrm{~mm}$ plate pressed onto the skin at the mastoid just behind the ear. The effectiveness of the sound transmission via BC is influenced by the anatomy and geometry of the head, which is changed by a mastoidectomy.

Mastoidectomy is a surgical procedure that removes the mastoid air cells of the temporal bone. It is performed to treat temporal bone ailments, such as cholesteatoma, but also for cochlear implantation (13). Two types of mastoidectomy are 1) closed, or canal wall up mastoidectomy (CWUM) and 2) open, or canal wall down mastoidectomy (CWDM) (13). During CWUM the external auditory canal is preserved, and in the case of cortical mastoidectomy so is the middle ear. CWDM results in the removal of the ear canal (posterior and superior canal walls). Two subtypes of CWDM are radical and modified radical mastoidectomy. Radical mastoidectomy converts the mastoid area, the middle ear, and the ear canal into a common cavity. The tympanic membrane, malleus and incus are removed, but the stapes footplate is kept intact. During the modified radical mastoidectomy, the mastoid area is exteriorized into the ear canal while preserving the middle ear (13). In the current work, two extreme cases are investigated: the least invasive, cortical mastoidectomy, and the most invasive, radical mastoidectomy (further referred to as CWUM and CWDM, respectively).

The hearing outcome of a mastoidectomy is often investigated by the air-bone gap, i.e. the difference in $A C$ and $B C$ hearing thresholds, shortly after the procedure (14) and as a long-term outcome (15). The AC sound is affected by the manipulations of the outer and middle ears, while the BC sound is affected by the manipulations of the middle ear $(4,16)$ and the sound transmission change due to the skull bone alterations. Consequently, the air-bone gap reveals combined effects on both $\mathrm{AC}$ and BC pathways.

A novel finite element model of the human head, the LiUHead (17), was used in this study. The LiUHead model is a computational tool that simulates vibrational patterns of a human head similar to experiments using cadaver heads and live patients $(1,18-24)$. The aim of the study is to investigate changes in BC sound transmission for two types of mastoidectomy (CWUM and CWDM) for two types of BC 
stimulation, one at the typical BAHA position directly at the skull bone and one at the skin surface at the mastoid, similar to a stimulation by a B71 transducer. 


\section{MATERIALS AND METHODS}

\section{Model overview}

All simulations were conducted in the whole head finite element model LiUHead, Fig. 1A. A detailed model description can be found in Chang, et al. (17). The model consists of eight domains - brain, cerebrospinal fluid (CSF), eyes, inner ears, cortical bone, diploë, cartilage, and soft tissue - and comprises a total of 87,100 nodes and 480,000 tetrahedral elements.

\section{Stimulation protocol}

Two stimulation devices at three locations on the right side of the head were simulated, Fig. 1B. First, a titanium screw $\varnothing 3 \times 5 \mathrm{~mm}$ was implanted in the skull at the standard location of the BAHA (6). The stimulation was 1 Newton harmonic force applied perpendicularly to the skull surface. Second, an acrylic plastic plate $\varnothing 15 \times 1.5 \mathrm{~mm}$, simulating the interface part of a Radioear B71 transducer, was placed flat on the surface of the mastoid skin. The stimulation was 1 Newton harmonic force applied at the transducer-skin interface, perpendicular to the skin surface. The B71 transducer was placed at 35 and $55 \mathrm{~mm}$ posterior to the ear canal opening, referred to as the standard and alternative positions, respectively. At the B71 alternative position, the transducer is entirely away from the area affected by the surgery. At the standard position, the transducer partially overlaps the affected area and is more affected by the surgery (Figs. 1C and 1D). The 4 Newton static force used to hold the B71 transducer in place was modelled by altering the mechanical properties of the soft tissue underneath the transducer plate so the resulting mechanical point impedance at the skin surface matched experimental data (25). The frequency range used in the simulations is from $100 \mathrm{~Hz}$ to $10,000 \mathrm{~Hz}$, with a resolution of $25 \mathrm{~Hz}$ between 100 and $500 \mathrm{~Hz}, 50 \mathrm{~Hz}$ between 550 and $1000 \mathrm{~Hz}$ and $200 \mathrm{~Hz}$ above 1,200 Hz.

\section{Mastoidectomy surgery}

Two types of mastoidectomies were simulated: CWUM and CWDM, Figs. 1C and 1D, respectively. The cavity in the skull created by the surgery can be left intact (without cavity obliteration) or filled with bone, cartilage or soft tissue (with cavity obliteration). In this work, the cavity obliteration is achieved using soft tissue. The soft tissue thickness at the location of the surgery is 10 to $20 \mathrm{~mm}$.

\section{Cochlear promontory vibration and the transcranial attenuation}

Cochlear promontory vibration is expressed as accelerance: the total (resultant) acceleration divided by the stimulating force. The total acceleration is calculated as the square root of the sum of the squares of the three orthogonal acceleration components (1). A slightly different method to calculate the resultant of a 3D vector has been proposed by Dobrev and Sim $(26,27)$. Our total acceleration was close to their maximum resultant magnitude, indicating the existence of a principal vibration direction.

Transcranial attenuation is a measure of the damping of the vibration from one side of the head to the other, here defined as the accelerance of the ipsilateral cochlea relative to the accelerance of the contralateral cochlea for a given stimulation position. 


\section{RESULTS}

\section{Cochlear promontory vibration}

The pre-mastoidectomy cochlear promontory accelerances are shown in Fig. 2. The accelerances from the ipsilateral and the contralateral cochleae show similar trends. The accelerance with the BAHA stimulation gradually increases with frequency below $2 \mathrm{kHz}$ and remains relatively flat above $2 \mathrm{kHz}$. The accelerances with the B71 stimulation increase with frequency at the low frequencies and peak at $1.5 \mathrm{kHz}$. At higher frequencies, the contralateral accelerance with the BAHA stimulation is $10 \mathrm{~dB}$ lower than the ipsilateral accelerance. The peak contralateral accelerance with the B71 stimulation is $5 \mathrm{~dB}$ below the peak ipsilateral accelerance, and falls more steeply with frequency than the ipsilateral at higher frequencies.

The post-mastoidectomy accelerance gain (Fig. 3 ) is defined as the ratio of post- to pre-mastoidectomy cochlear promontory accelerance. The ipsilateral gains with $\mathrm{B} 71$ stimulation are shown in Figs. $3 \mathrm{~A}$ and $3 \mathrm{~B}$, and with BAHA stimulation in Figs. $3 \mathrm{C}$ and $3 \mathrm{D}$. The contralateral gain with the $\mathrm{B} 71$ stimulation is shown in Figs. 3E and 3F, and with the BAHA stimulation in Figs. $3 \mathrm{G}$ and $3 \mathrm{H}$. The mastoidectomy caused an overall increase (positive gain) in the vibratory response of the cochlear promontory, especially at higher frequencies. The gain values are the highest on the ipsilateral side and with the B71 standard stimulation (Fig. 3B). The more invasive CWDM resulted in higher gains compared to CWUM, especially above $2 \mathrm{kHz}$ (Fig. 3B). Cavity obliteration caused between 1 and $3 \mathrm{~dB}$ lower accelerance gain with B71 stimulation, and less than $1 \mathrm{~dB}$ lower gain with BAHA stimulation. The ipsilateral gain with the B71 standard stimulation is below $4 \mathrm{~dB}$ for CWUM (Fig. 3A), but increases to $8 \mathrm{~dB}$ at the highest frequencies for CWDM (Fig. 3B). The gain with the B71 alternative stimulation follows the same trend as with the B71 standard stimulation, but with 1 to $2 \mathrm{~dB}$ lower values for both types of surgeries (Figs. $3 A$ and $B$ ). The ipsilateral gain with the BAHA stimulation increases with frequency to 4 and $6 \mathrm{~dB}$ for CWUM and CWDM, respectively (Figs. 3C and 3D).

The contralateral gains (Figs. $3 \mathrm{E}-\mathrm{H}$ ), similarly to the ipsilateral gains, are primarily positive. The values with B71 stimulation (Figs. 3E and 3F) are akin to the ipsilateral values (Figs. 3A and 3B). The gain with the BAHA stimulation (Figs. $3 \mathrm{G}$ and $3 \mathrm{H}$ ) peaks at $2 \mathrm{kHz}$ and drops to negative values at and above $8 \mathrm{kHz}$.

The difference in accelerance gains between CWUM and CWDM at the ipsilateral side is larger at higher frequencies with B71 standard stimulation (Fig. 4A) compared to BAHA stimulation (Fig. 4C), while the same difference at the contralateral side is $1 \mathrm{~dB}$ or less for all frequencies and types of stimulation (Figs. $4 B$ and $4 D$ ). With B71 stimulation at the ipsilateral cochlea the difference is close to $0 \mathrm{~dB}$ up to $2 \mathrm{kHz}$ and falls to $-5 \mathrm{~dB}$ and $-3 \mathrm{~dB}$ for the standard and alternative stimulation, respectively (Fig. $4 \mathrm{~A}$ ). The CWUM-CWDM difference at the ipsilateral cochlea with the BAHA stimulation (Fig. 4C) ranges from 0 to $-2 \mathrm{~dB}$. No effect from obliteration could be found when analyzing the results in Fig. 4, either for different stimulation positions or for ipsilateral or contralateral cochleae.

\section{Transcranial attenuation}


The transcranial attenuation, defined as the ratio of accelerances at the ipsilateral and contralateral cochlear promontories, for the pre-surgery condition is shown in Fig. 5A with B71 stimulation and in Fig. $5 B$ with BAHA stimulation. With the B71 standard stimulation, the attenuation is around $0 \mathrm{~dB}$ at frequencies below $400 \mathrm{~Hz}$ and increases with frequency to $18 \mathrm{~dB}$ at $10 \mathrm{kHz}$. The transcranial attenuation is similar to the $B 71$ standard and alternative stimulation for frequencies up to $4 \mathrm{kHz}$, above which the B71 alternative stimulation data show around $5 \mathrm{~dB}$ less attenuation (Fig. 5A). The transcranial attenuation with the BAHA stimulation remains around $0 \mathrm{~dB}$ at frequencies below $1 \mathrm{kHz}$ and plateaus around $10 \mathrm{~dB}$ at $2 \mathrm{kHz}$ and above (Fig. 5B).

The mastoidectomy effects on the transcranial attenuation are presented in Figs. $5 \mathrm{C}-\mathrm{F}$ as a difference between post- and pre-mastoidectomy transcranial attenuation. At frequencies below $2 \mathrm{kHz}$, the differences are less than $1 \mathrm{~dB}$ except with the BAHA stimulation and CWDM (Fig. 5F), where the difference reached $5 \mathrm{~dB}$ at $1 \mathrm{kHz}$. The transcranial attenuation difference after CWUM surgery with B71 stimulation (Fig. 5C) decreases above $2 \mathrm{kHz}$, reaching $-4 \mathrm{~dB}$ at $8 \mathrm{kHz}$. This is different for CWDM with $\mathrm{B} 71$ stimulation, where the difference is primarily positive at frequencies above $2 \mathrm{kHz}$ and reaches $5 \mathrm{~dB}$ at $10 \mathrm{kHz}$ (Fig. 5E). Influence of the exact stimulation position (standard or alternative) is observed only above $2 \mathrm{kHz}$ and is strongest with CWDM surgery. Obliteration does not influence the transcranial attenuation with B71 stimulation for the two surgical methods or the two stimulation positions (Figs. 5C and $5 \mathrm{E})$.

When the stimulation was at the BAHA position, there is a clear attenuation difference minimum of $-3 \mathrm{~dB}$ at $2 \mathrm{kHz}$ (Figs. 5D and 5F). This peak originates in the contralateral cochlear promontory vibration gain with the BAHA stimulation shown in Figs. $3 \mathrm{G}$ and $3 \mathrm{H}$. The transcranial attenuation post-pre difference with the BAHA stimulation is negative for both types of surgeries between 1.5 and $3 \mathrm{kHz}$, and primarily positive at frequencies above $3 \mathrm{kHz}$ (Figs. $5 \mathrm{D}$ and $5 \mathrm{~F}$ ). This can be interpreted as a mastoidectomy decreasing the transcranial attenuation at frequencies between 1.5 and $3 \mathrm{kHz}$, while it increases above this frequency range. Obliteration had nearly no impact on the transcranial attenuation difference with BAHA stimulation. 


\section{DISCUSSION}

\section{Cochlear promontory vibration}

The most common reason for mastoidectomy are mastoiditis, otitis media, and cholesteatoma (13). The surgery disrupts the normal AC hearing pathway (the middle ear and sometimes the ear canal is removed), resulting in a conductive hearing loss, and a BC hearing aid is often the best option to rehabilitate the patient (e.g. $6,7,8,28)$. The vibrations of the cochlear promontory are shown to be a reliable indicator of the hearing sensation $(1,18,20)$ and are used in this work as a quantitative indicator of the cochlear stimulation.

The results from Fig. 3 indicate that overall cochlear vibration level increases post-mastoidectomy. The increase is less than $5 \mathrm{~dB}$, except on the ipsilateral side for CWDM with B71 standard stimulation when the increase is $8 \mathrm{~dB}$ at $10 \mathrm{kHz}$ (Fig. 3B). The overall increase in vibration can be explained by reduced mass and stiffness of the temporal bone due to the surgery. Below $1 \mathrm{kHz}$, the head vibration resembles rigid body motion and reduced inertia leads to larger vibration levels (1). The reduced stiffness of the mastoid weakens the mastoid's attachment to the surrounding bone and leads to increased vibrations on the ipsilateral side.

The position of the stimulation device relative to the cochlea is important for $B C$ transmission (18). At the B71 standard position, the transducer is closest to the cochlea and the stimulation is directed towards the petrous part of the mastoid. The reduced mass and stiffness of the mastoid contribute to the cochlear stimulation when the stimulation is at the B71 standard position compared to B71 alternate and BAHA positions, which are further away from the cochlea and in the region where the skull bone is thinner compared to the B71 standard location. The stimulation at the thinner bone is less affected by the mastoidectomy since the flexing of the bone itself dominates the load impedance that determines the vibration transmitted to the skull bone. This is believed to be one of the explanations behind the differences found between the stimulation positions.

The two types of mastoidectomy result in similar vibration level changes of up to $4 \mathrm{~dB}$ at the cochlear promontory (Figs. 3 and 4). An exception is the B71 standard stimulation (Fig. 4A), where the cochlear promontory vibration for CWDM is up to $5 \mathrm{~dB}$ larger compared to CWUM. This is also reflected in the transcranial attenuation (Fig. 5), where the high-frequency attenuation is greater for CWDM than for CWUM when stimulation is applied with the B71 at the standard position. The transcranial attenuation is also greater at frequencies around $1 \mathrm{kHz}$ with CWDM than with CWUM when stimulation is with the BAHA. This post-CWDM increase in attenuation should not be interpreted as detrimental for unilaterally deaf subjects fitted with a $B C$ hearing aid, since the reason for the increase in attenuation is that the ipsilateral gain is greater than the contralateral gain (Fig. 3). Consequently, both types of surgery improve the contralateral excitation by 4 to $5 \mathrm{~dB}$ for frequencies between 1.5 and $6 \mathrm{kHz}$, improving the hearing rehabilitation for unilaterally deaf subjects fitted with a BC hearing aid.

Obliteration had a very week effect on the results, and the difference with and without obliteration is less than $1 \mathrm{~dB}$. The explanation is that the alteration of the mass and stiffness of the mastoid and the placement of the stimulation device have stronger effects on BC sound transmission. Soft tissue was 
used to obliterate the cavity in the simulations. It has lower stiffness than the bone and becomes decoupled from the surrounding bone at higher frequencies. Thus, replacing the bone with soft tissue in the process of cavity obliteration does not have a strong effect on the cochlear promontory vibration. However, if the obliteration forms new bone that fuses with the skull bone, the obliteration would probably reduce the cochlear vibration.

\section{Comparison with experimental findings}

In a recent study, Dobrev et al. (27) investigated the effect of mastoidectomy on BC sound by stimulating the skull bone at the BAHA position in cadaver heads and obtaining the cochlear promontory vibration with a 3D laser Doppler vibrometer (LDV) before and after CWUM. They report similar trends as in the present study up to $4 \mathrm{kHz}$, while above this frequency they report a decrease in the ipsilateral post-I pre-mastectomy gain and the current data indicate an increase in the same gain. One difference between the two studies is that Dobrev et al. (27) used the voltage to a piezo transducer as a reference, while the current study references to the force at the stimulation site. Another difference is that in the experimental data, slight manipulations of the ear are required for the laser beams to access the promontory pre-mastoidectomy, meaning that the head is not entirely intact. Also, the individual data in the Dobrev et al. (27) study are variable at the high frequency, with both positive and negative individual results.

\section{Clinical comparison and implications}

Most clinical studies of hearing outcome following different types of mastoidectomies report small or no differences between CWUM and CWDM $(14,29)$. According to the simulations presented here, a mastoidectomy is expected to result in an improvement of 2 to $5 \mathrm{~dB}$ at frequencies between 1 and $4 \mathrm{kHz}$, a frequency range often used for hearing threshold measurement. However, the greatest changes are expected at higher frequencies, above $4 \mathrm{kHz}$, which are not normally tested and can therefore go unnoticed. Varitiainen and Seppa (30) reported an average BC threshold improvement of $2.7 \mathrm{~dB}$ at $0.5 \mathrm{kHz}$, which declined with frequency to $0.2 \mathrm{~dB}$ at $4 \mathrm{kHz}$ after mastoidectomy. Similar results were reported by Choi, et al. (31), where mastoidectomy with intact ear canal improved the BC hearing threshold by on average $4 \mathrm{~dB}$, and with an open cavity the average improvement was $1 \mathrm{~dB}$. The $\mathrm{BC}$ hearing benefit with surgery declined with frequency, resulting in 2 to $3 \mathrm{~dB}$ worse improvement at $4 \mathrm{kHz}$. Vijayendra and Parikh (32) report an average improvement of 7.7 to $9.7 \mathrm{~dB}$, computed as a mean for the test frequencies between 0.5 and $4 \mathrm{kHz}$, after different types of mastoidectomies.

The results in these clinical studies show trends that are slightly different from the model predictions in Fig. 3. First, the general clinical trend is improved BC thresholds at the lower frequencies that decline with frequency, while the model simulations predict the opposite. Second, Choi et al. (32) found better threshold improvement with CWUM than CWDM, contrary to the data presented in Fig. 3 . There are several explanations for these differences. The clinical data are obtained from perceptual tests while the hearing thresholds from the model are based on promontory vibrations. Vibrations of the cochlear promontory have been reported as a reliable representation of the cochlear stimulation (20), but there can be subtle differences between cochlear promontory vibration and hearing thresholds. Also, the surgery itself can affect the hearing status. It has been suggested that the drill-induced noise can result 
in a permanent noise-induced hearing loss (33-35). Since the noise effect is prominent at higher frequencies, the improvement of the cochlear promontory vibrations at higher frequencies can be counteracted by a high frequency hearing loss. Also, the surgery may introduce scar tissue that results in thicker skin and soft tissue post-surgery. A stimulation on a thicker skin would mean a greater compliance between the B71 transducer and the skull, leading to a frequency-dependent reduction in the stimulation, similar to that reported in (31) and (32).

Yet another explanation for the differences seen is the manipulations of the middle ear. The LiUHead does not include a detailed middle ear, and the middle ear and mobility of the oval window have been indicated to influence BC hearing sensitivity $(4,36)$. However, the contribution of the middle ear inertia for $B C$ sound transmission has been shown to be about 10 to $20 \mathrm{~dB}$ below other $\mathrm{BC}$ contributors $(3,37,38)$. The combination of these factors is present in the clinical measurements of the hearing threshold and pre- to post-surgery comparison, while the LiUHead only incorporates the effects from the change in the skull anatomy and positioning of the $\mathrm{BC}$ device. Even so, the clinical results suggest a small but positive effect from the mastoidectomy, in line with the model prediction. Moreover, the greatest effect is suggested to appear at the highest frequencies, and BC hearing thresholds are clinically limited to $4 \mathrm{kHz}$ in most cases, excluding clinical data at frequencies where the greatest effect is predicted.

The results here for the BAHA position stimulation indicate a 3 to $5 \mathrm{~dB}$ improvement at the high frequencies after a mastoidectomy. This can be seen as a small and negligible effect. However, since one general limitation of $B C$ hearing aids is the limited maximum output level and dynamic range (39), a $5 \mathrm{~dB}$ improvement can be significant. Consequently, the $\mathrm{BC}$ hearing aid fitting range for people with mastoidectomies may be greater than for people with intact skulls.

\section{Conclusions}

Based on simulations in a finite element model of the human head (LiUHead), the post-surgery cochlear promontory vibration increases by up to 6 to $8 \mathrm{~dB}$, indicating an improvement in $\mathrm{BC}$ hearing sensitivity. The more invasive CWDM resulted in the largest sensitivity increase (vibration increase) on the ipsilateral side and with B71 stimulation. Contralateral side vibration response was largely unaffected by the type of surgery and gave up to $6 \mathrm{~dB}$ vibration increase. Below $2 \mathrm{kHz}$, the transcranial attenuation is relatively unaffected by the surgery type while at frequencies above $2 \mathrm{kHz}$, the attenuation increased by $5 \mathrm{~dB}$ after CWDM and decreased by a couple of dBs after CWUM. Cavity obliteration did not influence the results. 


\section{References}

1. Stenfelt S, Goode RL. Transmission properties of bone conducted sound: Measurements in cadaver heads. J Acoust Soc Am 2005;118:2373-91.

2. Stenfelt S. Acoustic and physiologic aspects of bone conduction hearing. Adv Otorhinolaryngol 2011;71:10-21.

3. Stenfelt S. Model predictions for bone conduction perception in the human. Hearing Res 2016;340:135-43.

4. Stenfelt S, Hato N, Goode RL. Factors contributing to bone conduction: The middle ear. J Acoust Soc Am 2002;111:947-59.

5. Stenfelt S, Wild T, Hato Net al. Factors contributing to bone conduction: The outer ear. J Acoust Soc Am 2003;113:902-13.

6. Tjellstrom A, Hakansson B, Granstrom G. Bone-anchored hearing aids - Current status in adults and children. Otolaryng Clin N Am 2001;34:337-+.

7. Bosman AJ, Snik AF, van der Pouw CTet al. Audiometric evaluation of bilaterally fitted boneanchored hearing aids. Audiology 2001;40:158-67.

8. Niparko JK, Cox KM, Lustig LR. Comparison of the bone anchored hearing aid implantable hearing device with contralateral routing of offside signal amplification in the rehabilitation of unilateral deafness. Otol Neurotol 2003;24:73-8.

9. Stenfelt $\mathrm{S}$, Zeitooni M. Binaural hearing ability with mastoid applied bilateral bone conduction stimulation in normal hearing subjects. J Acoust Soc Am 2013;134:481-93.

10. Priwin C, Stenfelt S, Granstrom Get al. Bilateral bone-anchored hearing aids (BAHAs): an audiometric evaluation. Laryngoscope 2004;114:77-84.

11. Zeitooni M, Maki-Torkko E, Stenfelt S. Binaural Hearing Ability With Bilateral Bone Conduction Stimulation in Subjects With Normal Hearing: Implications for Bone Conduction Hearing Aids. Ear Hearing 2016;37:690-702.

12. Reinfeldt $S$, Hakansson B, Taghavi Het al. New developments in bone-conduction hearing implants: a review. Med Devices (Auckl) 2015;8:79-93.

13. Fisch U, May J. Tympanoplasty, mastoidectomy, and stapes surgery. Stuttgart, New York: G. Thieme Verlag, Thieme Medical Publishers, 1994:xii, 292 p.

14. Kim MB, Choi J, Lee JKet al. Hearing Outcomes According to the Types of Mastoidectomy: A Comparison between Canal Wall Up and Canal Wall Down Mastoidectomy. Clin Exp Otorhinolar 2010;3:203-6.

15. Mukherjee P, Saunders N, Liu Ret al. Long-term outcome of modified radical mastoidectomy. J Laryngol Otol 2004;118:612-6.

16. Stenfelt S. Bone Conduction and the Middle Ear. In: Puria S, Popper A. N. and Fay R. F., ed. Springer Handbook of Auditory Research: The Middle Ear: Science, Otosurgery, and Technology. New York: Springer, 2012:135 - 69.

17. Chang $\mathrm{Y}, \mathrm{Kim} \mathrm{N}$, Stenfelt $\mathrm{S}$. The development of a whole-head human finite-element model for simulation of the transmission of bone-conducted sound. J Acoust Soc Am 2016;140:1635-51.

18. Eeg-Olofsson M, Stenfelt $\mathrm{S}$, Tjellstrom Aet al. Transmission of bone-conducted sound in the human skull measured by cochlear vibrations. Int J Audiol 2008;47:761-9.

19. Eeg-Olofsson M, Stenfelt S, Granstrom G. Implications for contralateral bone-conducted transmission as measured by cochlear vibrations. Otol Neurotol 2011;32:192-8.

20. Eeg-Olofsson M, Stenfelt S, Taghavi Het al. Transmission of bone conducted sound Correlation between hearing perception and cochlear vibration. Hearing Res 2013;306:1120.

21. Hakansson B, Carlsson P, Tjellstrom A. The Mechanical Point Impedance of the Human Head, with and without Skin Penetration. J Acoust Soc Am 1986;80:1065-75. 
22. Hakansson B, Eeg-Olofsson M, Reinfeldt Set al. Percutaneous versus transcutaneous bone conduction implant system: a feasibility study on a cadaver head. Otol Neurotol 2008;29:1132-9.

23. Stenfelt S. Transcranial Attenuation of Bone-Conducted Sound When Stimulation Is at the Mastoid and at the Bone Conduction Hearing Aid Position. Otol Neurotol 2012;33:105-14.

24. Stenfelt S, Goode RL. Bone-conducted sound: Physiological and clinical aspects. Otol Neurotol 2005;26:1245-61.

25. Cortés D. Bone Conduction Transducers: Output force dependency on load condition. In: Göteborg SCUoT, ed.2002.

26. Dobrev I, Sim JH. Magnitude and phase of three-dimensional (3D) velocity vector: Application to measurement of cochlear promontory motion during bone conduction sound transmission. Hear Res 2018;364:96-103.

27. Dobrev I, Sim JH, Pfiffner Fet al. Performance evaluation of a novel piezoelectric subcutaneous bone conduction device. Hearing Res 2018;370:94-104.

28. Snik AFM, Mylanus EAM, Proops DWet al. Consensus statements on the BAHA system: Where do we stand at present? Ann Oto Rhinol Laryn 2005;114:2-12.

29. Cook JA, Krishnan S, Fagan PA. Hearing results following modified radical versus canal-up mastoidectomy. Ann Otol Rhinol Laryngol 1996;105:379-83.

30. Vartiainen E, Seppa J. Results of bone conduction following surgery for chronic ear disease. Eur Arch Oto-Rhino-L 1997;254:384-6.

31. Choi HG, Lee DH, Chang KHet al. Frequency-Specific Hearing Results After Surgery for Chronic Ear Diseases. Clin Exp Otorhinolar 2011;4:126-30.

32. Vijayendra H, Parikh B. Bone Conduction Improvement After Surgery for Conductive Hearing Loss. Indian J Otolaryngol 2011;63:201-4.

33. Kylen P, Arlinger S. Drill-Generated Noise-Levels in Ear Surgery. Acta Oto-Laryngol 1976;82:402-9.

34. Abtahi SH, Fazel A, Rogha Met al. Effect of drill-induced noise on hearing in non-operated ear. Adv Biomed Res 2016;5:87.

35. Banakis Hartl RM, Mattingly JK, Greene NTet al. Drill-induced Cochlear Injury During Otologic Surgery: Intracochlear Pressure Evidence of Acoustic Trauma. Otol Neurotol 2017;38:938-47.

36. Stenfelt $\mathrm{S}$. Inner ear contribution to bone conduction hearing in the human. Hearing Res 2015;329:41-51.

37. Stenfelt $\mathrm{S}$. Middle ear ossicles motion at hearing thresholds with air conduction and bone conduction stimulation. J Acoust Soc Am 2006;119:2848-58.

38. Roosli C, Chhan D, Halpin Cet al. Comparison of umbo velocity in air- and bone-conduction. Hearing Res 2012;290:83-90.

39. van Barneveld DCPBM, Kok HJW, Noten JFPet al. Determining fitting ranges of various bone conduction hearing aids. Clin Otolaryngol 2018;43:68-75. 


\section{Figure legends}

Figure 1, Model overview. (A) An overview of the LiUHead and its components (CSF: cerebrospinal fluid). (B) Locations of the stimulation devices: the BAHA position, B71 stn (B71 standard position), and B71 alt (B71 alternative position). The BAHA position is in the skull bone while the B71 device was placed on the surface of the skin. (C). Canal wall up mastoidectomy (CWUM) and (D) canal wall down mastoidectomy (CWDM). The left column in panels $C$ and $D$ represents the $B 71$ standard position and the right column the B71 alternative position. Red area is the surgical cavity, dark yellow volume indicates the pressured tissue under the B71 transducer, and the dark blue plates are the B71 transducer interfacing the skin. The dark circle indicates the location of the ear canal opening.

Figure 2, Pre-mastoidectomy cochlear promontory accelerance level. Third-octave band averaged accelerance (total acceleration divided by stimulating force) from (A) ipsilateral and (B) contralateral cochlea with intact skull (pre-surgery). Stimulation at the BAHA position is indicated by a blue dotted line, stimulation at the B71 standard stimulation position by a black solid line, and stimulation at the B71 alternative stimulation position by a red dashed line.

Figure 3, Post-mastoidectomy accelerance gain. The gain is computed as the ratio of the thirdoctave band averaged post-mastoidectomy accelerance and the corresponding pre-mastoidectomy accelerance. Ipsilateral accelerance gain with B71 stimulation is shown for CWUM in (A) and for CWDM in (B). Ipsilateral accelerance gain with BAHA stimulation is shown for CWUM in (C) and for CWDM in (D). Contralateral accelerance gain with the B71 stimulation is shown for CWUM in (E) and for CWDM in $(\mathbf{F})$. Contralateral accelerance gain with the BAHA stimulation is shown for CWUM in (G) and for CWDM in $(\mathbf{H})$. Line properties are shown in the figure: stn and alt: B71 standard and alternative stimulation position, BAHA: BAHA stimulation position. Cavity obliteration is indicated by 'obl'.

Figure 4, Post-mastoidectomy CWUM-CWDM cochlear accelerance difference. The difference is computed by subtracting the third-octave band averaged accelerance gain (in $\mathrm{dB}$ ) after CWDM from the corresponding gain (in $\mathrm{dB}$ ) after CWUM. The difference with B71 stimulation at the ipsilateral cochlea is shown in (A) and at the contralateral cochlea in (B). Accelerance difference with BAHA stimulation at the ipsilateral cochlea is shown in (C) and at the contralateral cochlea in (D). Line properties are shown in the figure: stn and alt: B71 standard and alternative stimulation position; BAHA: BAHA stimulation position. Cavity obliteration is indicated by 'obl'.

Figure 5, Transcranial attenuation. Attenuation is computed as the ratio of the ipsilateral and contralateral cochlear promontory third-octave averaged accelerance values. Pre-mastoidectomy attenuation with B71 and stimulation is shown in (A) and with BAHA stimulation in (B). The difference between post- and pre-mastoidectomy transcranial attenuation after CWUM with the B71 stimulation is shown in (C) and with the BAHA stimulation in (D), and after CWDM with the B71 stimulation is shown in $(E)$ and with the BAHA stimulation in $(F)$. Line properties are shown in the figure: stn and alt: B71 standard and alternative stimulation position; BAHA: BAHA stimulation position. Cavity obliteration is indicated by 'obl'. 
A

B

Cortical bone

Soft tissue
Eye

Cartilage
C

CSF

Brain

Diploë

D

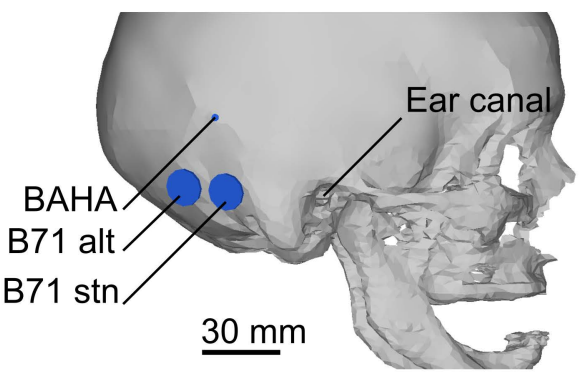

$20 \mathrm{~mm}$

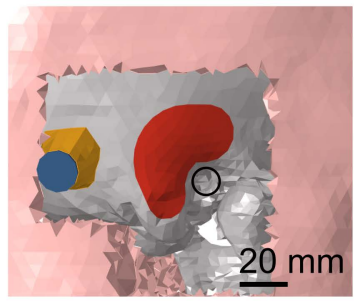

$20 \mathrm{~mm}$

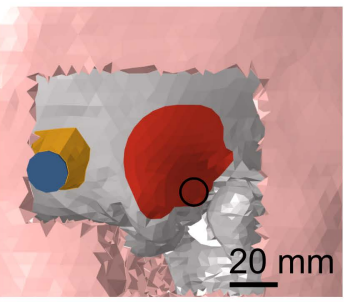

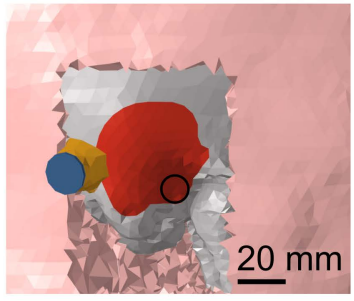



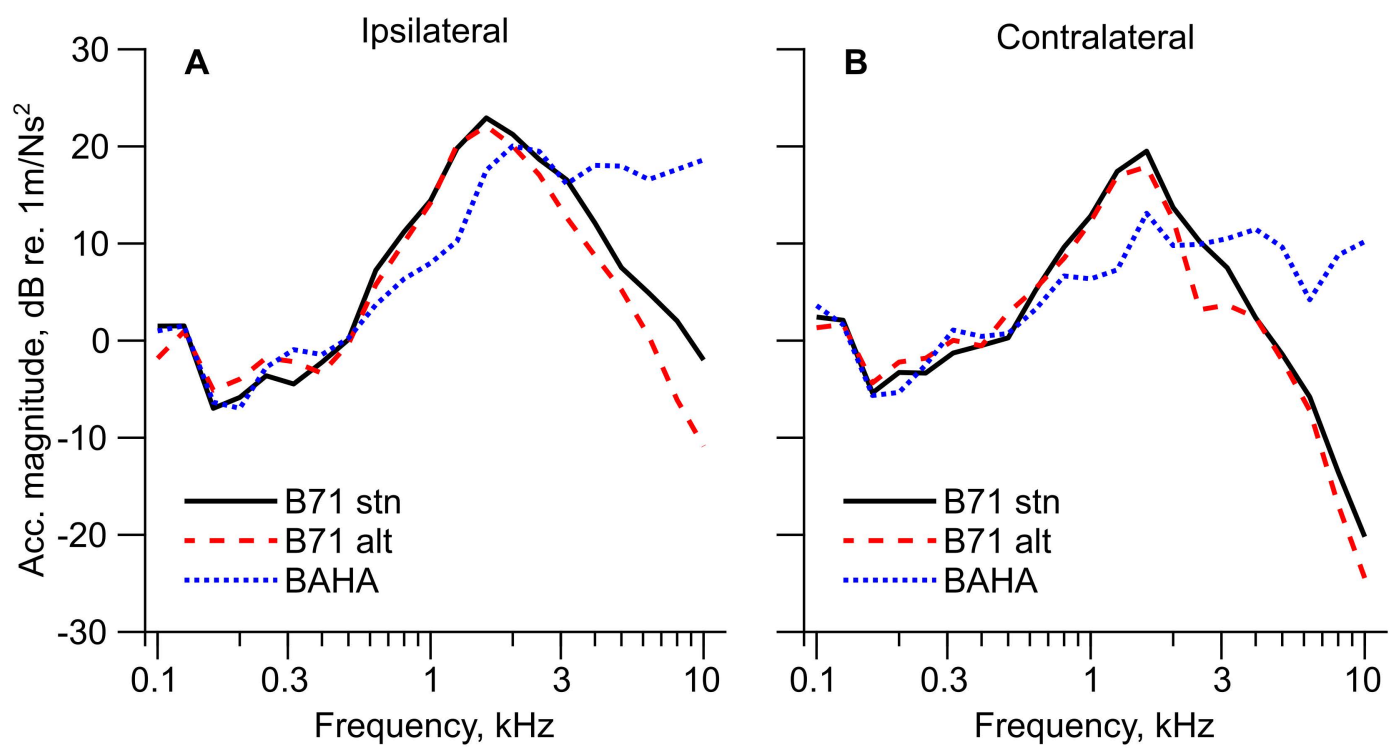

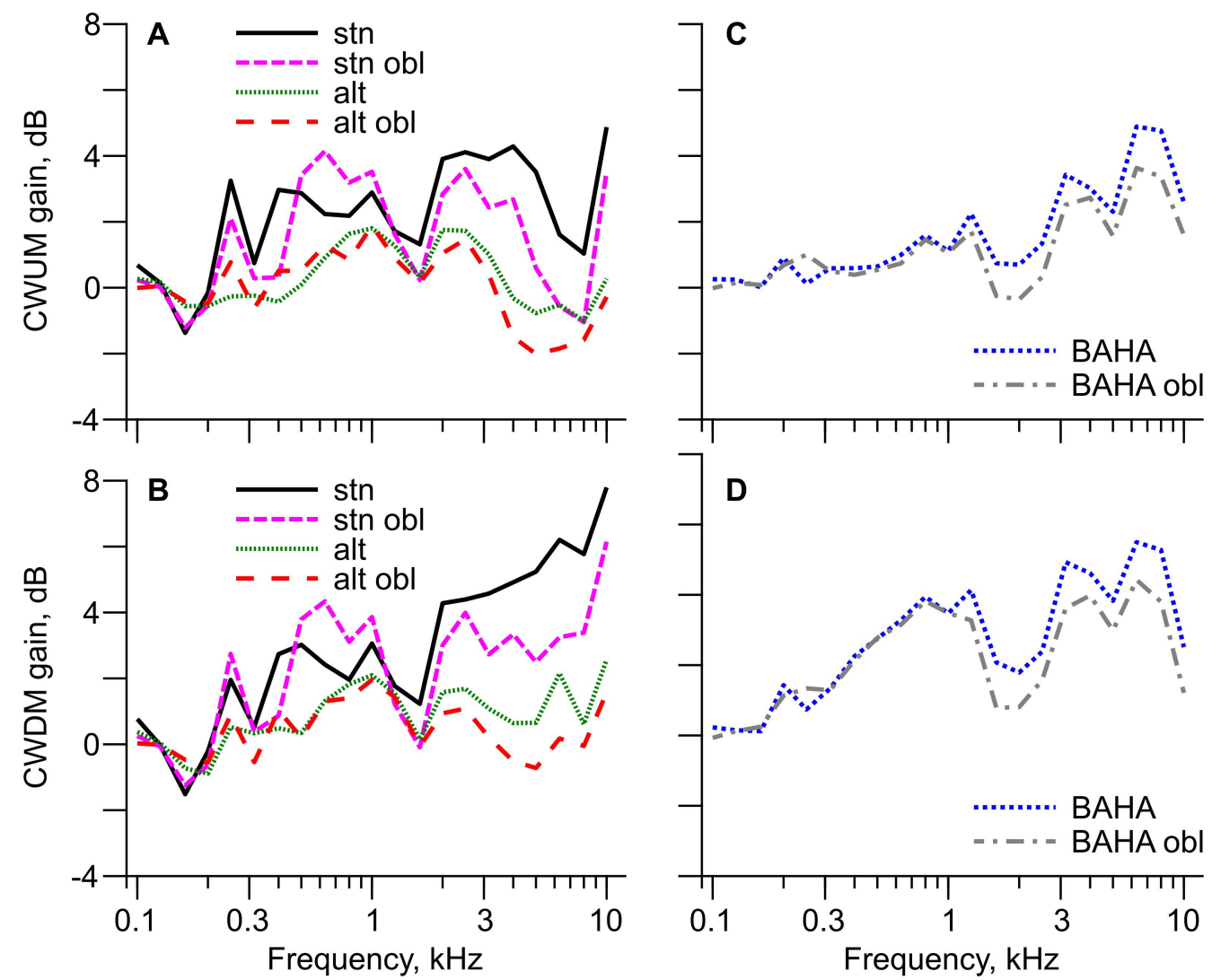

B71

Contralateral
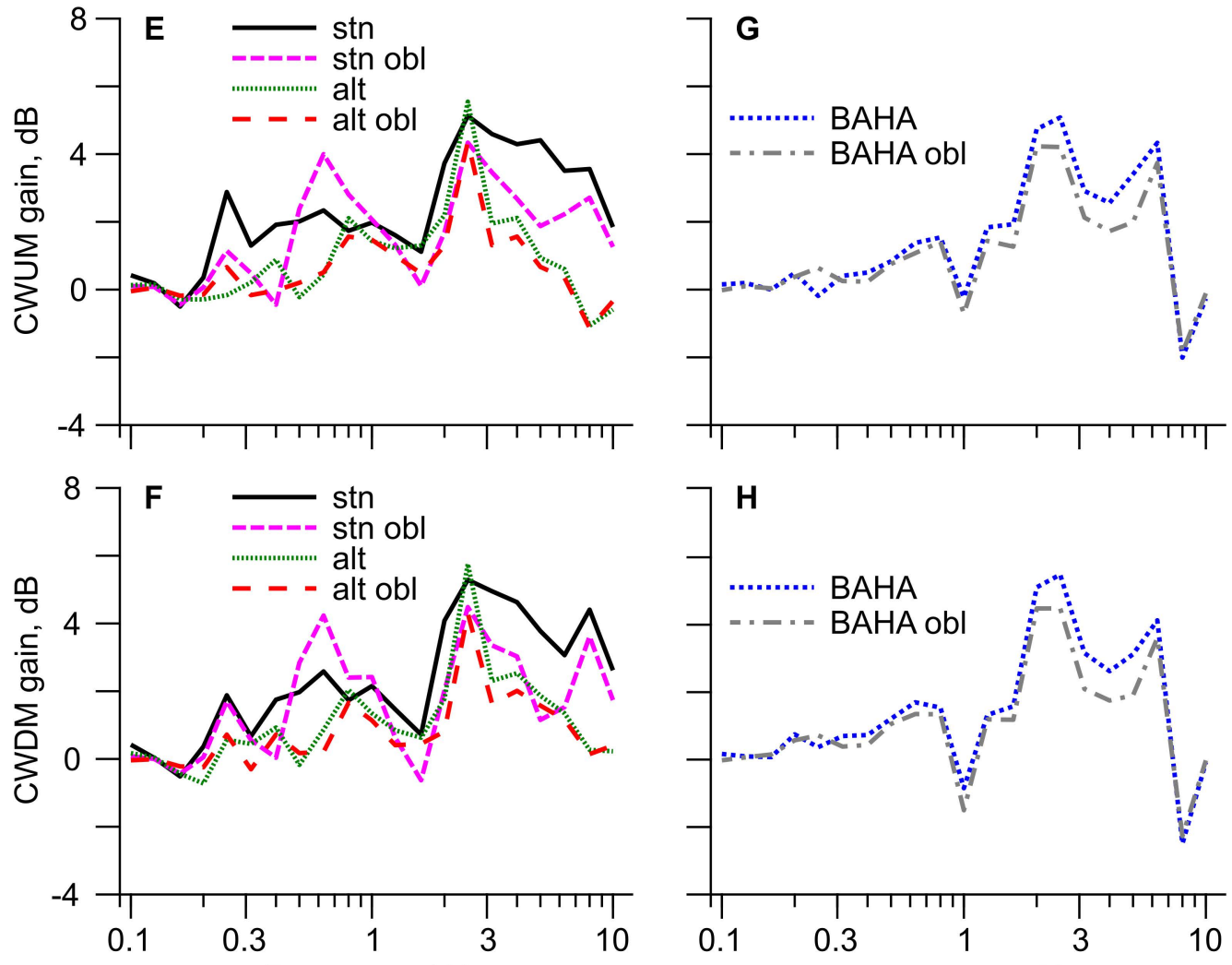

Frequency, kHz

Frequency, kHz 

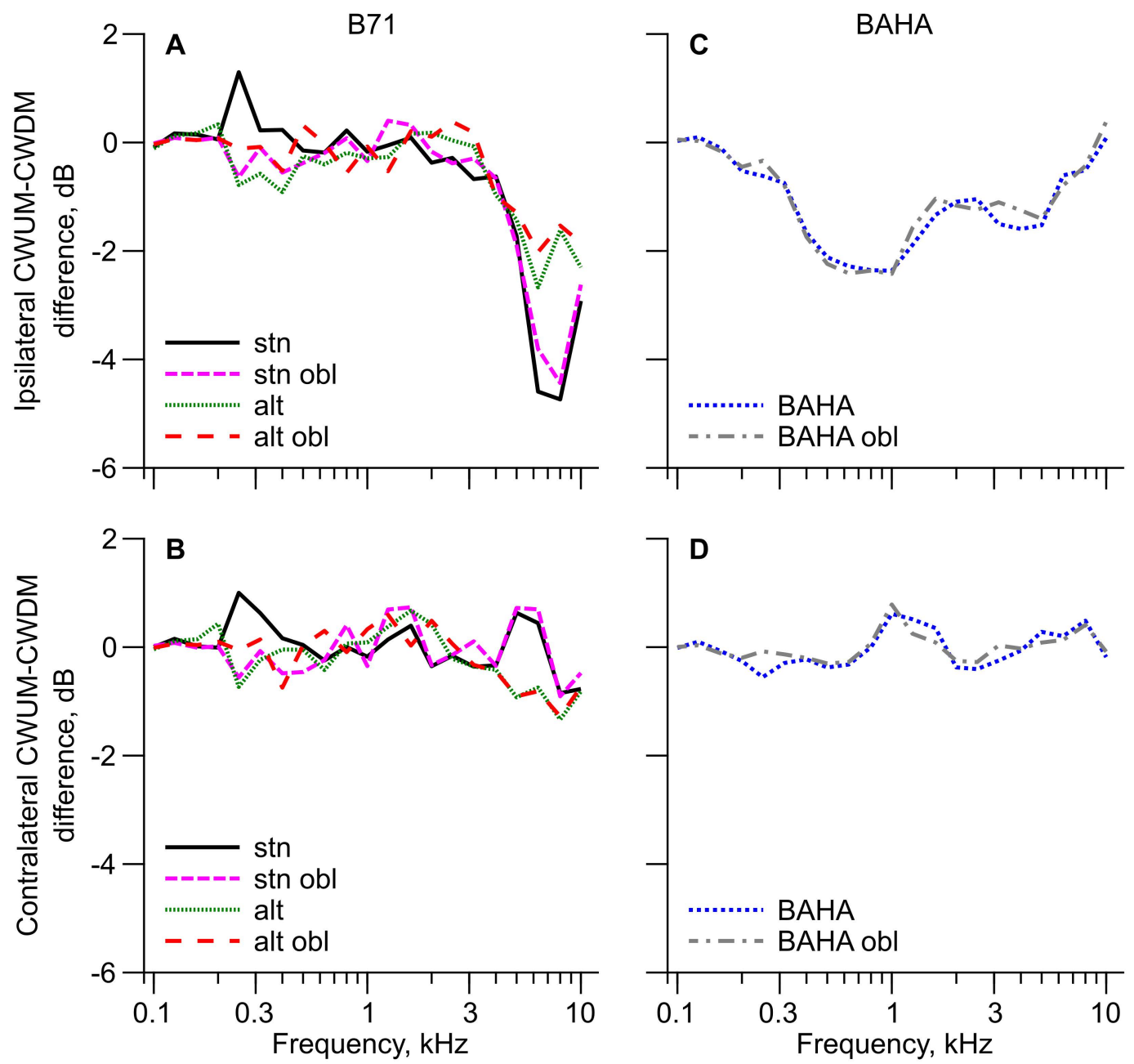

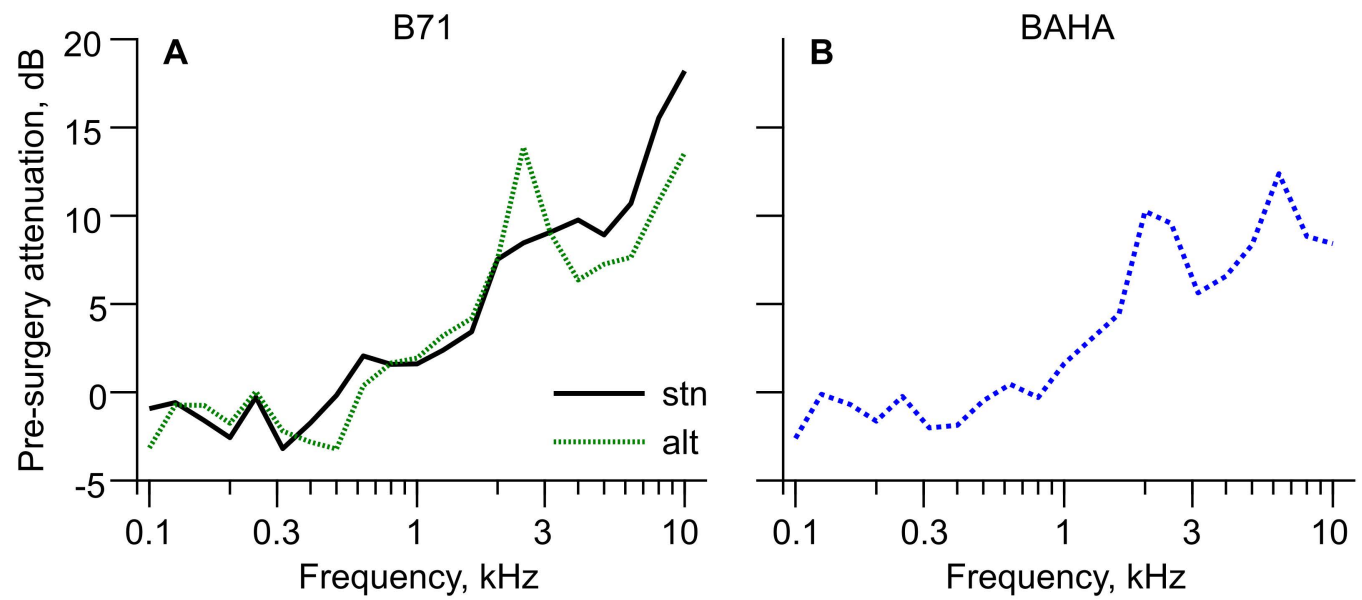

Difference between post- and pre-mastoidectomy
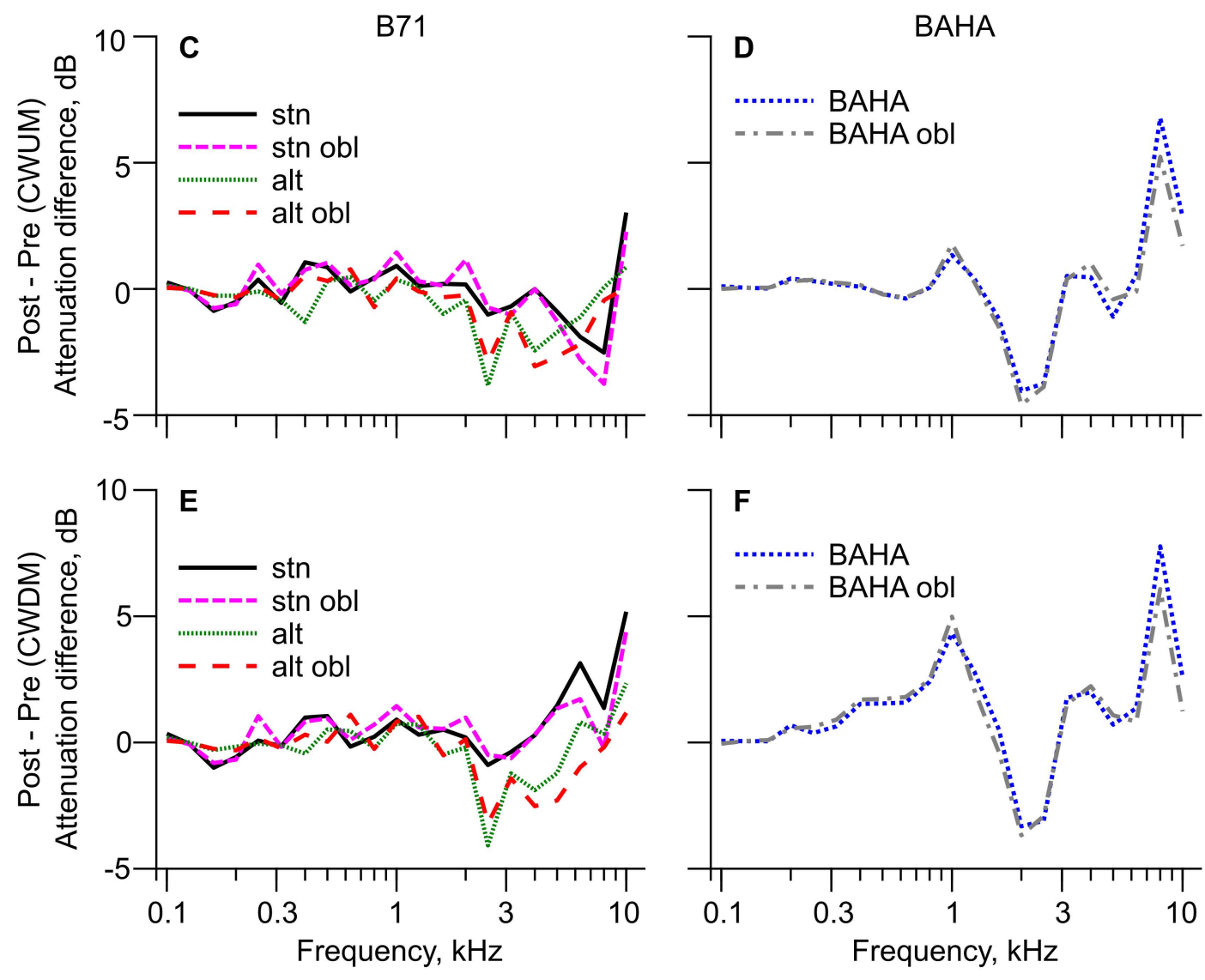